\title{
Integrated-optic dispersion compensator that uses chirped gratings
}

\author{
Cameron J. Brooks, Gerald L. Vossler, and Kim A. Winick \\ Department of Electrical Engineering and Computer Science, University of Michigan, Ann Arbor, Michigan 48109
}

Received October 20, 1994

\begin{abstract}
An integrated-optic dispersion compensator that uses chirped waveguide gratings is designed and fabricated. The 7-mm-long chirped grating is realized by a recently proposed method of curving a waveguide through a uniform grating. At $800 \mathrm{~nm}$, the fabricated device exhibits a reflection bandwidth in excess of $0.5 \mathrm{~nm}$ and a nearly quadratic phase response corresponding to a fiber dispersion-length product of $58 \mathrm{ps} / \mathrm{nm}$. The phase response reported is measured interferometrically with a narrow-band, tunable Ti:sapphire laser. Extending this technology to 5-cm-long gratings should permit dispersion compensation of 50-km-long fiber lengths at $1.55 \mu \mathrm{m}$.
\end{abstract}

The current trend in optical communication systems is toward higher bit rates and longer distances, where fiber chromatic dispersion becomes a performance-limiting factor. For systems operating at $1.5 \mu \mathrm{m}$ on standard telecommunicationsgrade optical fiber, the dispersion is approximately $20 \mathrm{ps} /(\mathrm{km} \mathrm{nm})$. Thus a 20-ps Gaussian pulse will expand to $60 \mathrm{ps}$ after it propagates through only $17 \mathrm{~km}$ of fiber. Therefore, correcting filters are needed that can compensate for the dispersion of the transmission fiber. These filters must have a frequency-dependent quadratic phase shift over a large enough frequency range to cover the bandwidth of the pulse. A variety of linear dispersion compensation devices have been proposed and demonstrated. These include Gires-Tournois interferometers, ${ }^{1}$ high-dispersion fibers, ${ }^{2}$ midpoint spectral inverters, ${ }^{3}$ integrated Mach-Zehnder interferometers, ${ }^{4}$ and chirped fiber gratings. ${ }^{5-7}$ There has been much interest lately in the use of fiber and waveguide grating filters for dispersion compensation, because these filters are relatively inexpensive, compact, and compatible with fiber-optic communication systems.

Fabrication of periodic gratings in fibers and planar waveguides is relatively easy. These structures, however, exhibit high dispersion only over small bandwidths. Ouellette ${ }^{8}$ proposed that this bandwidth limitation may be removed by use of a linearly chirped grating in place of a periodic structure. In such a device, the different frequency components in an incoming signal are Bragg matched at different points along the grating. Thus, on Bragg reflection, the signal traverses a path length that is frequency dependent. This frequency dependence results in either positive or negative dispersion, depending on the sign of the grating chirp.

There have been several recent reports of dispersion compensation using chirped gratings in fibers. ${ }^{5-7}$ Dispersion compensators that use chirped waveguide gratings on planar substrates have been proposed ${ }^{9}$; however, to the best of our knowledge, reports of such devices have not yet been published. The fabrication of these gratings can be quite challenging, because the required chirp is of the order of a few parts in ten thousand. One method for achieving such small chirps in a controlled fashion is to taper the width of a waveguide that contains a uniform grating. ${ }^{10}$ Alternatively, an arbitrary chirp can be obtained by bending a waveguide through a uniform grating. ${ }^{11}$ The functional form of the chirp is determined by the curve shape of the waveguide, and this can be controlled easily. In this Letter we report on the design, fabrication, and measurement of an integrated, chirped waveguide filter capable of dispersion compensation. ${ }^{12}$ The linearly chirped grating is realized by etching a uniform grating into a curved, ion-exchanged glass waveguide.

The period $\Lambda(z)$ of a linearly chirped grating can be written as

$$
\Lambda(z)=\Lambda_{0}+\frac{\Lambda_{0}^{2} F z}{\pi L_{g}^{2}}
$$

where $\Lambda_{0}$ is the nominal grating period, $L_{g}$ is the grating length, $z$ is the distance measured along the grating, and $F$ represents a dimensionless chirp parameter. The complex reflection coefficient, $r(\omega)$, corresponding to the grating described by Eq. (1) can be determined by numerically solving a standard pair of coupled-mode equations for the field amplitudes. ${ }^{13}$ When $F>(\sim 100), r(\omega)$ is given approximately by

$$
\begin{aligned}
r(\omega)= & \exp \left\{i \left[C_{0}+C_{1}\left(\omega-\omega_{0}\right)+\frac{L_{g}^{2} N_{\text {eff }}^{2}}{F c^{2}}\right.\right. \\
& \left.\left.\times\left(\omega-\omega_{0}\right)^{2}\right]\right\}
\end{aligned}
$$

where $N_{\text {eff }}$ is the effective index of the guided mode, $c$ is the speed of light in vacuum, $\omega_{0}$ is the Bragg frequency corresponding to grating period $\Lambda_{0}, \omega$ is the optical frequency, and $C_{0}$ and $C_{1}$ are constants. The linear and constant phase terms result only in a time delay and do not affect the performance of the dispersion-compensating filter. The filter bandwidth, $\Delta \omega$, is approximately

$$
\frac{\Delta \omega}{\omega_{0}} \approx \frac{\Lambda_{0} F}{\pi L_{g}}
$$


and should be at least as large as the transmitted bit rate. If this filter is to provide dispersion compensation for a fiber length $L_{f}$, then it follows from Eq. (2) that $F$ and $L_{g}$ must be chosen to satisfy

$$
\frac{\lambda_{0}^{2}}{4 \pi c} D L_{f}=-\frac{L_{g}^{2} N_{\mathrm{eff}}^{2}}{F c^{2}}
$$

where $D$ is the fiber dispersion in units of seconds per square meter.

It is easy to see that the effective period of a grating can be chirped by etching a uniform grating into a curved waveguide. If the uniform grating has period $\Lambda_{0}$, then the effective grating period at position $z$ is given by $\Lambda_{0}\left\{1+[\mathrm{d} y(z) / \mathrm{d} z]^{2}\right\}^{1 / 2}$, where $y(z)$ is the curve that describes the waveguide bend. For small chirps, the equation of the waveguide curve corresponding to Eq. (1) can be derived by simple geometry:

$$
y(z)=\frac{2}{3} z^{3 / 2}\left(2 \frac{\Lambda_{0} F}{\pi L_{g}^{2}}\right)^{1 / 2} .
$$

Thus the amount of chirp can be precisely controlled by specifying the appropriate curve design. It is also important to note that the bandwidth of these filters is proportional to $F / L_{g}$. Therefore $F$ and $L_{g}$ must be chosen so that the filter exhibits sufficiently large bandwidth in addition to the desired dispersion.

For demonstration purposes, a dispersion filter was designed to compensate for a fiber dispersion-length product, $D L_{f}$ of $65 \mathrm{ps} / \mathrm{nm}$ at $800 \mathrm{~nm}$. Such a filter could recompress a dispersion-broadened 16-ps (FWHM) pulse back to its 4-ps transform limit. We chose a grating length $L_{g}=7 \mathrm{~mm}$ and a chirp $F=$ 112 , which can be shown from relations (3) and (4) to yield the desired dispersion-length product and have adequate bandwidth to pass a 4-ps-wide pulse. The reflection coefficient of the filter was calculated by solving the coupled mode equations numerically with $\kappa L_{g}=3 \pi$ ( $\kappa$ is the grating coupling coefficient). Figure 1 shows the reflectance and the phase of this filter as functions of the normalized frequency deviation $\left(\delta L_{g}\right)$ :

$$
\delta=\frac{2 \pi}{\lambda} N_{\text {eff }}-\frac{\pi}{\Lambda_{0}} .
$$

The filter has a bandwidth of approximately $1.1 \mathrm{~nm}$, and the phase very closely approximates a quadratic in the central region of this response. Over the plotted region, the phase deviation from a quadratic is less than $0.4 \mathrm{rad}$. When a dispersion-broadened 16-ps Gaussian pulse is applied to this filter, the resulting output is a nearly transform-limited recompressed 4-ps pulse, as shown in Fig. 2.

Based on this design, the integrated dispersioncompensation device, shown in Fig. 3, was fabricated. First, channel waveguides were formed in soda-lime glass by $\mathrm{Ag}^{+}$-ion exchange $(0.2 \mathrm{wt} . \%$ $\mathrm{AgNO}_{3}$ and 99.8 wt. $\% \mathrm{NaNO}_{3}$ ) for 8 min at $340{ }^{\circ} \mathrm{C}$. The exchange was performed through a $4-\mu \mathrm{m}$-wide curved waveguide diffusion mask. The waveguide curve shape was given by Eq. (5) with $L_{g}=7 \mathrm{~mm}$,
$F=112$, and $\Lambda_{0}=0.265 \mu \mathrm{m}$. The curve had a maximum slope of $3^{\circ}$. A grating period of approximately $0.265 \mu \mathrm{m}$ was chosen to facilitate testing with an 800-nm Ti:sapphire laser. First-order gratings were holographically exposed in a $180-\mathrm{nm}$ layer of photoresist on top of the sample by a HeCd laser. Following development, the grating was argon-ion milled into the waveguide to a depth of approximately $60 \mathrm{~nm}$.

The optical characteristics of the device were measured with a tunable, narrow-linewidth Ti:sapphire laser. Figure 4 shows the reflected TE power measured from the dispersion compensator. Devices with etched gratings of this type generally have lower TM reflectances, but this polarization sensitivity could be removed by overcoating the device with an $\mathrm{SiO}_{2}$ layer. Investigations are currently under way to determine the source of the ripples in

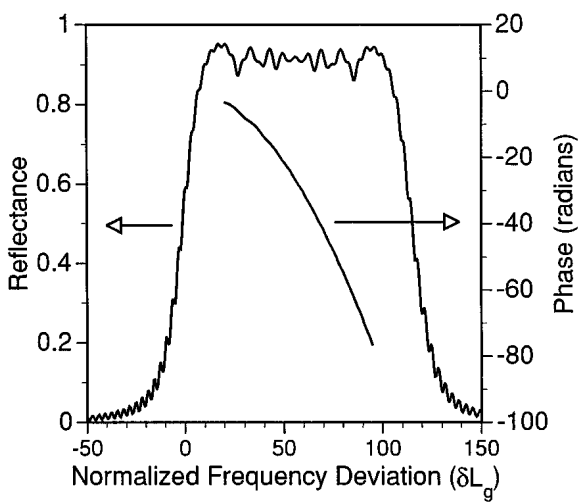

Fig. 1. Reflectance and phase of a linearly chirped grating filter $\left(\kappa L_{g}=3 \pi, F=112\right)$.

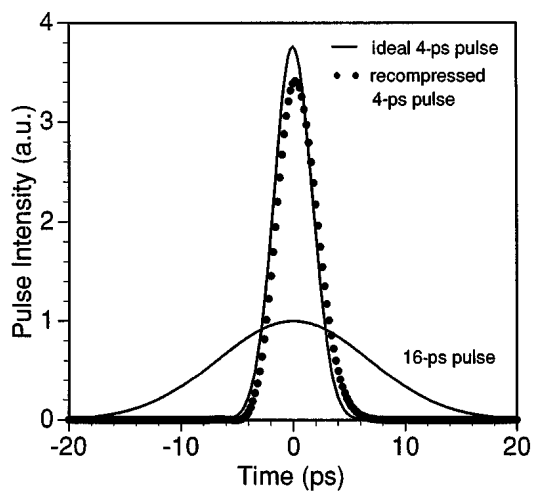

Fig. 2. Performance of the linearly chirped grating filter $\left(\kappa L_{g}=3 \pi, F=112\right)$ as a pulse compressor.

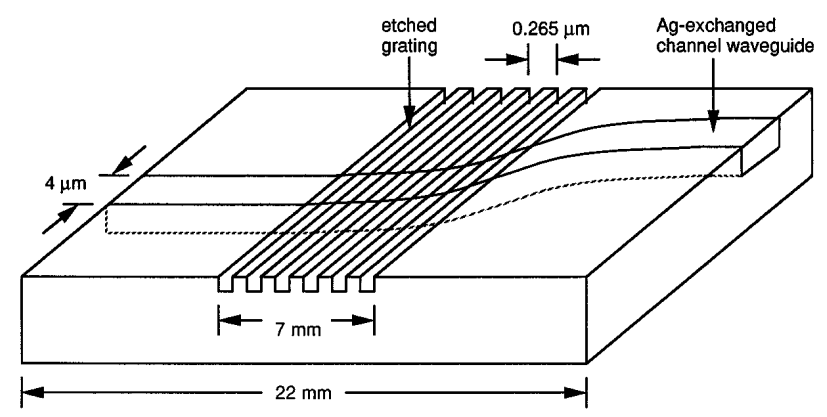

Fig. 3. Integrated dispersion compensator. 


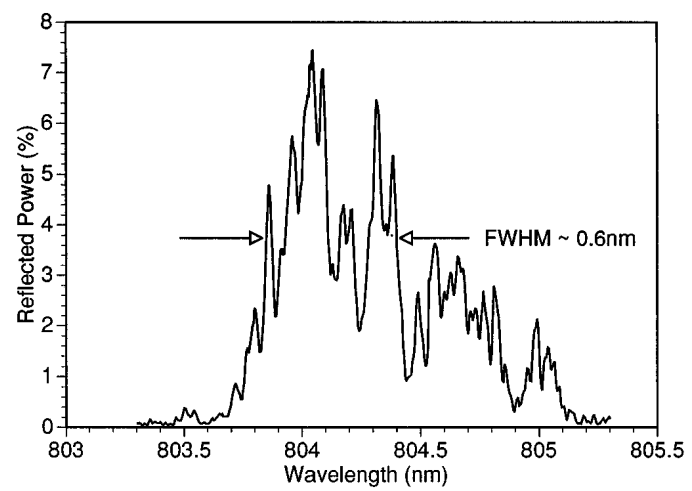

Fig. 4. Measured reflectance of the dispersion compensator.

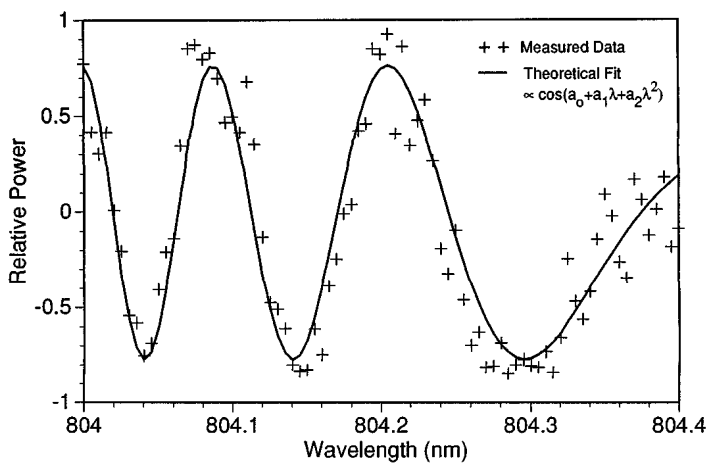

Fig. 5. Interferometric phase measurement of the dispersion compensator.

the reflected response; however, numerical calculations indicate that similar reflection characteristics can be obtained from filters with relatively low coupling coefficients. Larger coupling coefficients could be obtained by varying the grating and ion-exchange parameters. It is evident, however, that this device exhibits significant reflected power over a bandwidth of approximately $0.6 \mathrm{~nm}$, which is more than enough to accommodate a 4 -ps pulse.

The phase response of our device was measured interferometrically with the tunable Ti:sapphire laser mentioned above. A Michelson interferometer was built to interfere light reflected from the dispersion compensator with light from a reference path to create fringes. A small portion of a fringe was imaged onto a detector, and its power, $P(\lambda)$, was measured as the Ti:sapphire laser was tuned. The detected power can be described by

$$
P(\lambda)=P_{1}+P_{2}(\lambda)+2 \alpha \sqrt{P_{1} P_{2}(\lambda)} \cos [\phi(\lambda)] .
$$

Here $P_{1}$ is the power from the reference path, $P_{2}(\lambda)$ is the reflected power from the dispersion compensator, and $\phi(\lambda)$ is the phase difference between the two paths. The constant, $\alpha<1$, appears in the expression because the detector collects power over some finite region of a single fringe. For an ideal dispersion compensator, the phase should be a quadratic in $\lambda$, that is,

$$
\phi(\lambda)=2 \pi\left(a_{0}+a_{1} \lambda+a_{2} \lambda^{2}\right) .
$$

It follows from Eq. (8) that the dispersion, in picoseconds per nanometer, is given by

$$
\frac{2 \pi c}{\lambda_{0}^{2}} \frac{\mathrm{d}^{2} \phi}{\mathrm{d} \omega^{2}}=\frac{6 \lambda_{0}^{2}}{\pi c} a_{2} .
$$

$P(\lambda), P_{1}$, and $P_{2}(\lambda)$ were measured, and then the data were numerically fitted to Eqs. (7) and (8). The result is shown in Fig. 5. The value of $a_{2}$ for this fit was $14.05 \mathrm{~nm}^{-2}$, which corresponds to a measured dispersion of $58.0 \mathrm{ps} / \mathrm{nm}$. This value is in good agreement with the theoretically desired dispersion of $65 \mathrm{ps} / \mathrm{nm}$.

In summary, we have designed and built the first (to the best of our knowledge) integrated dispersion compensator based on a chirped grating realized by use of a curved waveguide. The phase response of the device was measured and agrees with the theoretical predictions. The design technique is flexible and could be applied to meet the requirements of a variety of communications systems. If the grating length were extended to $5 \mathrm{~cm}$ (with $F=100$ ), for example, this device could compensate for the dispersion of $50 \mathrm{~km}$ of standard fiber at $1.55 \mu \mathrm{m}$, operating at data rates of tens of gigabits per second or less.

This work was supported in part by the National Science Foundation under grants ECS-9201079 and STC PHY 8920108 through the Center for Ultrafast Optical Sciences and by IMRA America, Inc.

\section{References}

1. A. H. Gnauck, C. R. Giles, L. J. Cimini, Jr., J. Stone, L. W. Stulz, S. K. Korotty, and J. J. Veselka, IEEE Photon. Technol. Lett. 3, 1147 (1991).

2. C. D. Poole, J. M. Wiesenfeld, and D. J. DiGiovanni, IEEE Photon. Technol. Lett. 5, 194 (1993).

3. R. M. Jopson, A. H. Gnauck, and R. M. Derosier, Electron. Lett. 29, 576 (1993).

4. K. Takiguchi, K. Okamoto, S. Suzuki, and Y. Ohmori, IEEE Photon. Technol. Lett. 6, 86 (1994).

5. K. O. Hill, F. Bilodeau, B. Malo, T. Kitagawa, S. Theriault, D. C. Johnson, J. Albert, and K. Takiguchi, Opt. Lett. 19, 1314 (1994).

6. B. J. Eggleton, P. A. Krug, L. Poladian, K. A. Ahmed, and H.-F. Liu, Opt. Lett. 19, 877 (1994).

7. R. Kashyap, S. V. Chernikov, P. F. McKee, and J. R. Taylor, Electron. Lett. 30, 1078 (1994).

8. F. Ouellette, Opt. Lett. 12, 847 (1987).

9. J. E. Roman and K. A. Winick, IEEE J. Quantum Electron. 29, 975 (1993)

10. K. C. Byron, K. Sugden, T. Bricheno, and I. Bennion, Electron. Lett. 29, 1659 (1993).

11. H. Hillmer, K. Magari, and Y. Suzuki, IEEE Photon. Technol. Lett. 5, 10 (1993).

12. C. J. Brooks, G. L. Vossler, and K. A. Winick, in Digest of Optical Society of America Annual Meeting (Optical Society of America, Washington, D.C., 1994), paper MT4.

13. T. Tamir, ed., Guided Wave Optoelectronics (SpringerVerlag, New York, 1988), Chap. 2. 